\title{
Chemoenzymatic Synthesis of Sacranosides A and B
}

\author{
Eiji Kawahara, ${ }^{a, b}$ Mikio FunII, ${ }^{c}$ Yoshiteru IDA, ${ }^{c}$ and Hiroyuki AkitA ${ }^{*, a}$ \\ ${ }^{a}$ School of Pharmaceutical Sciences, Toho University; 2-2-1 Miyama, Funabashi, Chiba 274-8510, Japan: ${ }^{b}$ Tsukuba \\ Research Institute, Novartis Pharma K.K.; 8 Ohkubo, Tsukuba, Ibaraki 300-2611, Japan: and ${ }^{c}$ School of Pharmaceutical \\ Sciences, Showa University; 1-5-8 Hatanodai, Shinagawa-ku, Tokyo 142-8555, Japan. \\ Received October 5, 2005; accepted November 29, 2005
}

Direct $\beta$-glucosidation between (-)-myrtenol and nerol and D-glucose (3) using the immobilized $\beta$-glucosidase from almonds with the synthetic prepolymer ENTP-4000 gave myrtenyl $O$ - $\beta$-D-glucoside (4) and neryl $O$ - $\beta$ D-glucoside (10), respectively. The coupling of the myrtenyl or neryl $O$ - $\beta$-D-glucopyranoside congeners (7 or 13) and 2,3,4-tri- $O$-benzoyl- $\beta$-L-arabinopyranosyl bromide (8) afforded the coupled products $(9$ or 14$)$, respectively. Deprotection of the coupled products $(9$ or 14 ) afforded the synthetic myrtenyl 6- $O$ - $\alpha$-L-arabinopyranosyl- $\beta$-Dglucopyranoside (Sacranoside A, 1) or neryl 6-O- $\alpha$-L-arabinopyranosyl- $\beta$-D-glucopyranoside (Sacranoside B, 2), respectively.

Key words $\beta$-glucosidase; $\beta$-glucosidation; natural product synthesis; Sacranoside

Monoterpene glycosides are a group of water soluble natural products widely distributed in the plant kingdom. ${ }^{1)}$ The biological activity of some compounds has been determined and has been reported to indicate antibacterial activity, cytotoxic and antioxidant properties, enzyme inhibition, and immunomodulatory properties. ${ }^{2-5)}$ Among them, two kinds of naturally occurring monoterpene alcohol $6-O$-glycosy- $\beta$-Dglucopyranoside congeners, myrtenyl 6- $O-\alpha$-L-arabinopyranosyl- $\beta$-D-glucopyranoside (Sacranoside A, 1) and neryl 6$O$ - $\alpha$-L-arabinopyranosyl- $\beta$-D-glucopyranoside (Sacranoside $\mathrm{B}, 2)$ were isolated from a methanol extract of Rhodiola sacra (Prain ex HAMET) S. H. Fu (Crassulaceae). ${ }^{2)}$ Synthesis of these $\beta$-D-glucopyranoside congeners aroused our interest for the purpose of investigating their pharmacological activity. In this paper, we describe the total synthesis of naturally occurring 1 and 2 based on the selective $\beta$-glycosidation catalyzed by the immobilized $\beta$-glucosidase (EC 3.2.1.21) from almonds between D-glucose (3) and monoterpene alcohol.

Enzymatic $\boldsymbol{\beta}$-Glycosidation In direct $\beta$-glycosidation between D-glucose (3) and primary alcohols using $\beta$-glucosidase (EC 3.2.1.21) from almonds under thermodynamic conditions, a high concentration of alcohol or a medium with low water activity is reported to be effective. ${ }^{6)}$ On the other hand, we reported the effectiveness of immobilization of $\beta$ glucosidase (EC 3.2.1.21) from almonds with a photocrosslinkable resin prepolymer (ENTP-4000) in the direct $\beta$-glucosidation between D-glucose (3) and 1,8-octanediol. ${ }^{7)}$ We then examined the direct $\beta$-glucosidation between D-glucose (3) and monoterpene alcohols using the reported immobilized $\beta$-glucosidase (EC 3.2.1.21) ${ }^{7}$ from almonds. When a large amount of (-)-myrtenol and nerol (19.2 eq) was used as an acceptor for D-glucose (3) in the presence of the immobilized $\beta$-glucosidase, $4.2 \%$ yield of myrtenyl $O-\beta$-D-glucopyranoside (4) and 7.2\% yield of neryl $O$ - $\beta$-D-glucopyra- noside (10) were obtained, respectively.

Synthesis of Myrtenyl 6- $O$ - $\alpha$-L-Arabinopyranosyl- $\beta$-Dglucopyranoside (Sacranoside A, 1) tert-Butyldimethylsilylation of $\mathbf{4}$ gave a silyl ether $(\mathbf{5} ; 57 \%$ yield), which was subjected to benzoylation to give a benzoate (6) in $98 \%$ yield. Desilylation of 6 using $1 \mathrm{~N} \mathrm{HCl}$ provided the desired 7 in $79 \%$ yield. By applying the reported procedure, ${ }^{8)}$ coupling reaction of myrtenyl $\beta$-D-glucopyranoside congener (7) and $2,3,4$-tri- $O$-benzoyl- $\beta$-L-arabinopyranosyl bromide $(\mathbf{8})^{9)}$ in the presence of silver triflate (AgOTf) and tetramethylurea (TMU) gave the corresponding coupling product (9) in $74 \%$ yield. Finally, treatment of $\mathbf{9}$ with $\mathrm{NaOMe}$ in $\mathrm{MeOH}$ provided the synthetic myrtenyl $6-O-\alpha$-L-arabinopyranosyl- $\beta$-Dglucopyranoside (1) in $44 \%$ yield. The spectral data $\left({ }^{13} \mathrm{C}\right.$ and $\left.{ }^{1} \mathrm{H}-\mathrm{NMR}\right)$ and optical rotation $\left\{[\alpha]_{\mathrm{D}}^{28}-52.2^{\circ}(c=1.55\right.$, $\mathrm{MeOH})\}$ of the synthetic (1) were identical to those of natural product $1\left\{[\alpha]_{\mathrm{D}}^{25}-33.9^{\circ}(c=0.1, \mathrm{MeOH})\right\}^{2)}$

Synthesis of Neryl 6- $O$ - $\alpha$-L-Arabinopyranosyl- $\beta$-D-glucopyranoside (Sacranoside B, 2) tert-Butyldimethylsilylation of $\mathbf{1 0}$ gave a silyl ether $(\mathbf{1 1} ; 80 \%$ yield), which was subjected to benzoylation to give a benzoate (12) in $98 \%$ yield. Desilylation of $\mathbf{1 2}$ using $1 \mathrm{~N} \mathrm{HCl}$ provided the desired $\mathbf{1 3}$ in $93 \%$ yield. The coupling reaction of neryl $\beta$-D-glucopyranoside congener (13) and $\mathbf{8}$ in the presence of AgOTf and TMU gave the coupled product (14) in $96 \%$ yield. Finally, treatment of 14 with $\mathrm{NaOMe}$ in $\mathrm{MeOH}$ provided the synthetic neryl 6- $O-\alpha$-L-arabinopyranosyl- $\beta$-D-glucopyranoside (2) in $48 \%$ yield. The spectral data $\left({ }^{13} \mathrm{C}\right.$ - and $\left.{ }^{1} \mathrm{H}-\mathrm{NMR}\right)$ of the synthetic 2 were consistent with those of natural product $\mathbf{2}$, while optical rotation of synthetic $2\left\{[\alpha]_{\mathrm{D}}^{26}-34.9^{\circ}(c=1.85\right.$, $\mathrm{MeOH})\}$ were found to be different from the reported data $\left\{[\alpha]_{\mathrm{D}}^{25}+11.6^{\circ}(c=0.3, \mathrm{MeOH})\right\}^{2,10)}$
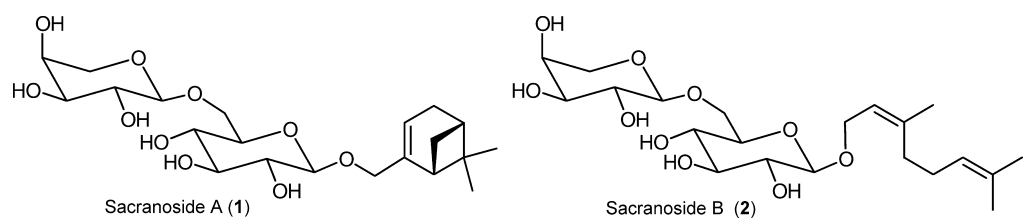

Chart 1 


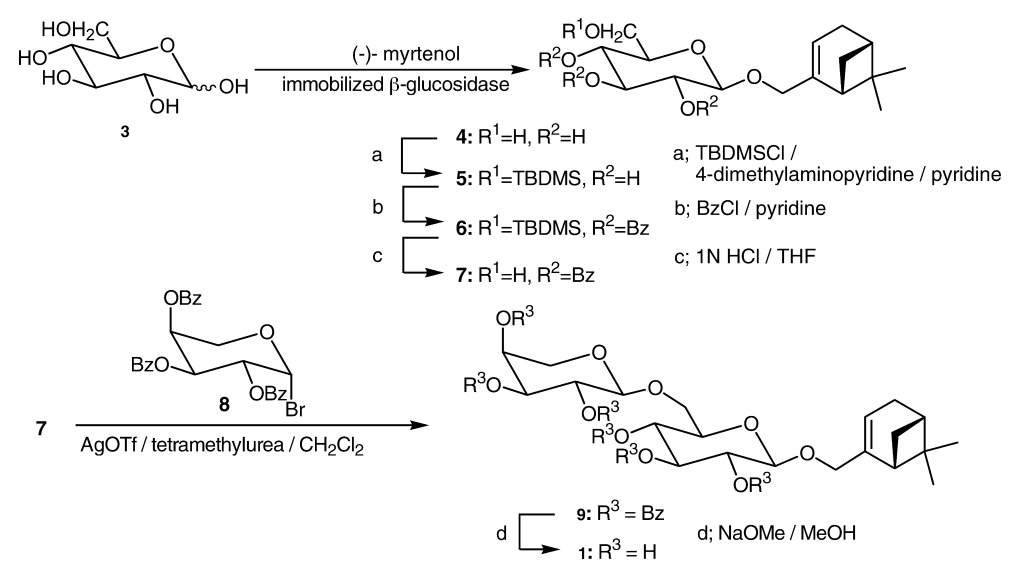

Chart 2

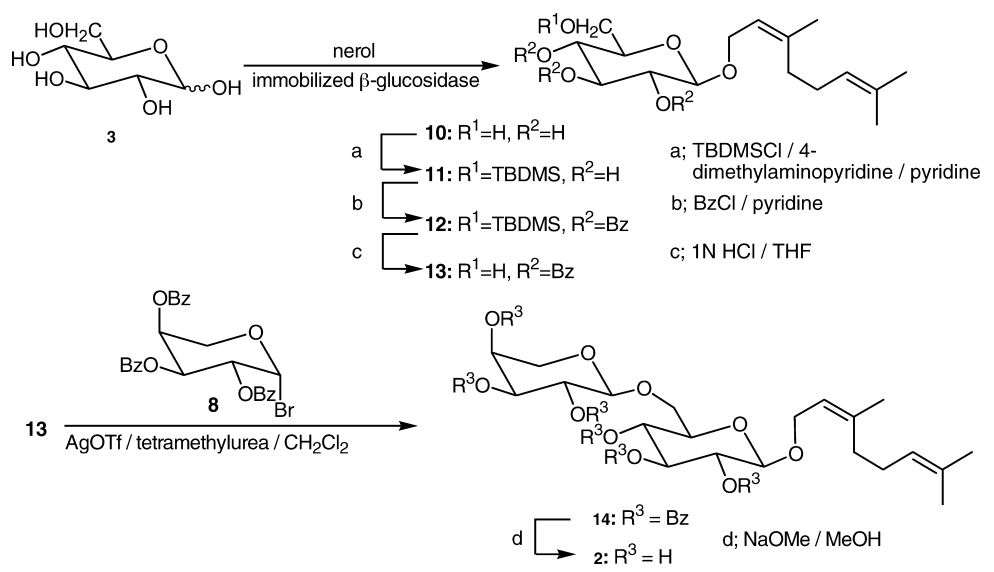

Chart 3

\section{Conclusion}

In conclusion, direct $\beta$-glucosidation between (-)myrtenol and nerol and D-glucose (3) using the immobilized $\beta$-glucosidase from almonds with the synthetic prepolymer ENTP-4000 gave myrtenyl $O$ - $\beta$-D-glucoside (4) in $4.2 \%$ yield and neryl $O$ - $\beta$-D-glucoside (10) in $7.2 \%$ yield, respectively. The coupling of the myrtenyl or neryl $O$ - $\beta$-D-glucopyranoside congeners $(\mathbf{7}, \mathbf{1 3})$ and $2,3,4$-tri- $O$-benzoyl- $\beta$-L-arabinopyranosyl bromide (8) afforded the coupled products $(9$ or 14), respectively. Deprotection of the coupled products (9 or 14) afforded the synthetic myrtenyl 6- $O-\alpha$-L-arabinopyranosyl- $\beta$-D-glucopyranoside (Sacranoside A, 1) or neryl 6- $O$ $\alpha$-L-arabinopyranosyl- $\beta$-D-glucopyranoside (Sacranoside B, 2), respectively.

\section{Experimental}

${ }^{1} \mathrm{H}$ - and ${ }^{13} \mathrm{C}$-NMR spectra were recorded on a BRUKER AV400M spectrometer or JEOL AL 400 spectrometer. Spectra were recorded with 5$10 \%$ (w/v) solution in $\mathrm{CDCl}_{3}$ or $\mathrm{CD}_{3} \mathrm{OD}$ with $\mathrm{Me}_{4} \mathrm{Si}$ as an internal reference. High-resolution mass spectra (HR-MS) and the fast atom bombardment mass spectra (FAB-MS) were obtained with a JEOL JMS 600H spectrometer. Optical rotations were measured on a JASCO DIP-370 digital polarimeter. IR spectra were recorded on a JASCO FT/IR-300 spectrophotometer. Al reagents were purchased from commercial sources and used without purification. All evaporations were performed under reduced pressure. For column chromatography, silica gel (Kieselgel 60) was employed for flash column chromatography and silica gel (silica gel 60N, spherical, neutral, 40$50 \mathrm{~mm}$ ) was employed.

Immobilization of $\boldsymbol{\beta}$-D-Glucosidase Using a Prepolymer $\beta$-D-Glucosidase (EC 3.2.1.21) from almonds was purchased from Sigma Chemical Co.
(G-0395, 2.5-3.6 U/mg). Immobilization of this $\beta$-D-glucosidase on the photocross-linkable resin prepolymer (ENTP-4000) was carried out using the following procedure. One gram of ENTP- 4000 was mixed with $10 \mathrm{mg}$ of a photosensitizer, benzoin ethyl ether, and $110 \mathrm{mg}$ of $\beta$-D-glucosidase from almonds $(3.4 \mathrm{U} / \mathrm{mg})$. The mixture was layered on a sheet of transparent polyester film (thickness, $c a .0 .5 \mathrm{~mm}$ ). The layer was covered with transparent thin film and then illuminated with chemical lamps (wavelength range, $300-400 \mathrm{~nm}$ ) for $3 \mathrm{~min}$. The gel film thus obtained was cut into small pieces $(0.5 \times 5 \times 5 \mathrm{~mm})$ and used for the next reaction.

Enzymatic Synthesis of Myrtenyl $\boldsymbol{O}$ - $\boldsymbol{\beta}$-D-Glucopyranoside (4) A mixture of D-glucose (3) $(1.1 \mathrm{~g}, 6.1 \mathrm{mmol})$, myrtenol $(18.0 \mathrm{~g}, 118 \mathrm{mmol})$, water $(2 \mathrm{ml})$, and the immobilized $\beta$-glucosidase $(1.1 \mathrm{~g})$ was incubated for $4 \mathrm{~d}$ at $50^{\circ} \mathrm{C}$. The reaction mixture was filtered off and the filtrate was directly chromatographed on silica gel $(35 \mathrm{~g})$ to give myrtenyl $O$ - $\beta$-D-glucopyranoside $(4,81 \mathrm{mg}, 0.26 \mathrm{mmol}, 4.2 \%)$ as a white solid from the $\mathrm{CHCl}_{3} / \mathrm{MeOH}=10: 1$ eluent. 4: $[\alpha]_{\mathrm{D}}^{29}-56.6^{\circ}(c=1.2, \mathrm{MeOH})$; IR $(\mathrm{KBr})$ : $3393,2916,1369,1076,1030 \mathrm{~cm}^{-1} ;{ }^{1} \mathrm{H}-\mathrm{NMR}\left(\mathrm{CD}_{3} \mathrm{OD}\right): \delta 0.87(3 \mathrm{H}, \mathrm{s})$, $1.19(1 \mathrm{H}, \mathrm{d}, J=8.6 \mathrm{~Hz}), 1.30(3 \mathrm{H}, \mathrm{s}), 2.07-2.13(1 \mathrm{H}, \mathrm{m}), 2.22-2.26(1 \mathrm{H}$ m), $2.27-2.32(2 \mathrm{H}, \mathrm{m}), 2.43(1 \mathrm{H}, \mathrm{ddd}, J=5.6,5.6,8.6 \mathrm{~Hz}), 3.19(1 \mathrm{H}, \mathrm{dd}$, $J=7.6,8.5 \mathrm{~Hz}), 3.21-3.24(1 \mathrm{H}, \mathrm{m}), 3.26-3.30(1 \mathrm{H}, \mathrm{m}), 3.33-3.36(1 \mathrm{H}$, $\mathrm{m}), 3.66(1 \mathrm{H}, \mathrm{dd}, J=2.3,11.9 \mathrm{~Hz}), 3.86(1 \mathrm{H}, \mathrm{dd}, J=2.3,11.9 \mathrm{~Hz}), 3.98-$ $4.03(1 \mathrm{H}, \mathrm{m}), 4.20-4.24(1 \mathrm{H}, \mathrm{m}), 4.28(1 \mathrm{H}, \mathrm{d}, J=7.6 \mathrm{~Hz}), 5.55-5.58(1 \mathrm{H}$, $\mathrm{m}) ;{ }^{13} \mathrm{C}-\mathrm{NMR}\left(\mathrm{CD}_{3} \mathrm{OD}\right): \delta 21.5,26.6,32.2,32.5,38.9,42.2,44.6,62.8$, $71.7,72.7,75.1,77.9,78.2,103.4,120.8,146.3$; HR-FAB-MS [ $m$-nitrobenzyl alcohol (NBA)] $m / z$ : Calcd for $\mathrm{C}_{16} \mathrm{H}_{27} \mathrm{O}_{6}: 315.1808(\mathrm{M}+1)^{+}$, Found: 315.1803 .

Enzymatic Synthesis of Neryl $\boldsymbol{O}$ - $\boldsymbol{\beta}$-D-Glucopyranoside (10) A mixture of D-glucose $(3)(1.1 \mathrm{~g}, 6.1 \mathrm{mmol})$, nerol $(18.0 \mathrm{~g}, 117 \mathrm{mmol})$, water $(2 \mathrm{ml})$, and the immobilized $\beta$-glucosidase $(1.1 \mathrm{~g})$ was incubated for $4 \mathrm{~d}$ at $50{ }^{\circ} \mathrm{C}$. The reaction mixture was filtered off and the filtrate was directly chromatographed on silica gel $(35 \mathrm{~g})$ to give neryl $O$ - $\beta$-D-glucopyranoside $(\mathbf{1 0}, 139 \mathrm{mg}, \quad 0.44 \mathrm{mmol}, 7.2 \%)$ as a white solid from the $\mathrm{CHCl}_{3} / \mathrm{MeOH}=10: 1$ eluent. 10: $[\alpha]_{\mathrm{D}}^{29}-18.9^{\circ}(c=0.49, \mathrm{MeOH})$; IR $(\mathrm{KBr})$ : 
3408, 2920, 1079, $1042 \mathrm{~cm}^{-1} ;{ }^{1} \mathrm{H}-\mathrm{NMR}\left(\mathrm{CD}_{3} \mathrm{OD}\right): \delta 1.61(3 \mathrm{H}, \mathrm{s}), 1.67(3 \mathrm{H}$, s), $1.75(3 \mathrm{H}, \mathrm{s}), 2.06-2.19(4 \mathrm{H}, \mathrm{m}), 3.17(1 \mathrm{H}, \mathrm{dd}, J=7.8,7.8 \mathrm{~Hz}), 3.20-$ $3.25(1 \mathrm{H}, \mathrm{m}), 3.26-3.36(2 \mathrm{H}, \mathrm{m}), 3.67(1 \mathrm{H}, \mathrm{dd}, J=5.3,11.9 \mathrm{~Hz}), 3.85(1 \mathrm{H}$, $\mathrm{dd}, J=2.0,11.9 \mathrm{~Hz}), 4.21(1 \mathrm{H}, \mathrm{dd}, J=7.8,12.0 \mathrm{~Hz}), 4.27(1 \mathrm{H}, \mathrm{d}, J=7.8 \mathrm{~Hz})$, $4.32(1 \mathrm{H}, \mathrm{dd}, J=6.3,12.0 \mathrm{~Hz}), 5.09-5.16(1 \mathrm{H}, \mathrm{m}), 5.35-5.40(1 \mathrm{H}, \mathrm{m})$; ${ }^{13} \mathrm{C}-\mathrm{NMR}\left(\mathrm{CD}_{3} \mathrm{OD}\right): \delta 17.8,23.7,25.9,27.7,33.1,62.7,66.3,71.7,75.1$, $77.9,78.2,102.9,122.6,125.0,132.8,141.8$; HR-FAB-MS (NBA) $\mathrm{m} / \mathrm{z}$ : Calcd for $\mathrm{C}_{16} \mathrm{H}_{29} \mathrm{O}_{6}: 317.1965(\mathrm{M}+1)^{+}$, Found: 317.1978 .

Myrtenyl 6-O-tert-Butyldimethylsilyl- $\boldsymbol{\beta}$-D-glucopyranoside (5) A mixture of 4 (700 mg, $2.05 \mathrm{mmol}$ ), 4-dimethylaminopyridine (DMAP; $6 \mathrm{mg}$, $0.05 \mathrm{mmol})$ and TBDMSCl $(352 \mathrm{mg}, 2.34 \mathrm{mmol})$ in pyridine $(15 \mathrm{ml})$ was stirred for $16 \mathrm{~h}$ at $\mathrm{rt}$. The reaction mixture was evaporated under reduced pressure to give a residue, which was purified by flash column chromatography on silica gel $\{30 \mathrm{~g}, n$-hexane/AcOEt $(4: 1)-\mathrm{AcOEt}\}$ to afford $5(541 \mathrm{mg}$, $1.26 \mathrm{mmol}, 57 \%)$ as a colorless syrup. 5: $[\alpha]_{\mathrm{D}}^{29}-50.9^{\circ}\left(c=2.2, \mathrm{CHCl}_{3}\right)$; IR (KBr): 3433, 2927, 1473, 1253, $1048 \mathrm{~cm}^{-1}$; ${ }^{1} \mathrm{H}-\mathrm{NMR}\left(\mathrm{CDCl}_{3}\right): 0.10(3 \mathrm{H}, \mathrm{s})$, $0.11(3 \mathrm{H}, \mathrm{s}), 0.84(3 \mathrm{H}, \mathrm{s}), 0.91(9 \mathrm{H}, \mathrm{s}), 1.18(1 \mathrm{H}, \mathrm{d}, J=8.6 \mathrm{~Hz}), 1.28(3 \mathrm{H}, \mathrm{s})$, $2.01-2.12(1 \mathrm{H}, \mathrm{m}), 2.13-2.17(1 \mathrm{H}, \mathrm{m}), 2.22-2.34(2 \mathrm{H}, \mathrm{m}), 2.40(1 \mathrm{H}$, ddd, $J=5.6,5.6,8.6 \mathrm{~Hz}), 3.33-3.41(2 \mathrm{H}, \mathrm{m}), 3.55-3.60(2 \mathrm{H}, \mathrm{m}), 3.83$ $(1 \mathrm{H}, \mathrm{dd}, J=6.3,10.4 \mathrm{~Hz}), 3.93(1 \mathrm{H}, \mathrm{dd}, J=5.1,10.4 \mathrm{~Hz}), 3.97-3.99(1 \mathrm{H}$, m), $4.18-4.23(1 \mathrm{H}, \mathrm{m}), 4.31(1 \mathrm{H}, \mathrm{d}, J=7.6 \mathrm{~Hz}), 5.53-5.55(1 \mathrm{H}, \mathrm{m}) ;{ }^{13} \mathrm{C}-$ NMR $\left(\mathrm{CDCl}_{3}\right): \delta-4.99[2 \mathrm{C}], 18.7,21.7,26.4[3 \mathrm{C}], 26.7,31.8,32.1,38.5$, $41.3,44.0,65.4,72.4,73.7,74.1,74.5,76.7,101.8,121.3,144.7$; HR-FABMS (NBA) $m / z$ : Calcd for $\mathrm{C}_{22} \mathrm{H}_{41} \mathrm{O}_{6} \mathrm{Si}: 429.2672(\mathrm{M}+1)^{+}$, Found: 429.2630 .

Myrtenyl 2,3,4-Tri- $O$-benzoyl-6-O-tert-butyldimethylsilyl- $\beta$-D-glucopyranoside (6) To a solution of $5(450 \mathrm{mg}, 1.05 \mathrm{mmol})$ in pyridine $(10 \mathrm{ml})$ was added benzoyl chloride $(738 \mathrm{mg}, 5.25 \mathrm{mmol})$ at $0{ }^{\circ} \mathrm{C}$, and the whole was stirred for $14 \mathrm{~h}$ at $\mathrm{rt}$. The reaction mixture was diluted with water and extracted with AcOEt. The organic layer was washed with $0.5 \mathrm{~N} \mathrm{HCl}$, $\mathrm{H}_{2} \mathrm{O}$ and brine. Then the organic layer was dried over $\mathrm{Na}_{2} \mathrm{SO}_{4}$ and evaporated to give a residue, which was purified by flash column chromatography on silica gel $\{30 \mathrm{~g}, n$-hexane/AcOEt $(8: 1-4: 1)\}$ to afford $6(759 \mathrm{mg}$, $1.02 \mathrm{mmol}, 98 \%)$ as a colorless syrup. 6: $[\alpha]_{\mathrm{D}}^{29}-24.2^{\circ}\left(c=0.59, \mathrm{CHCl}_{3}\right)$; IR (KBr): 2929, 2359, 1733, 1262, $1091 \mathrm{~cm}^{-1} ;{ }^{1} \mathrm{H}-\mathrm{NMR}\left(\mathrm{CDCl}_{3}\right): \delta 0.03(6 \mathrm{H}$, s), $0.77(3 \mathrm{H}, \mathrm{s}), 0.87(9 \mathrm{H}, \mathrm{s}), 1.02(3 \mathrm{H}, \mathrm{s}), 1.05(1 \mathrm{H}, \mathrm{d}, J=8.6 \mathrm{~Hz}), 1.93-$ $2.03(2 \mathrm{H}, \mathrm{m}), 2.14(1 \mathrm{H}, \mathrm{ddd}, J=5.6,5.6,8.6 \mathrm{~Hz}), 2.10-2.25(2 \mathrm{H}, \mathrm{m})$, $3.79-3.86(3 \mathrm{H}, \mathrm{m}), 3.98-4.05(1 \mathrm{H}, \mathrm{m}), 4.23-4.28(1 \mathrm{H}, \mathrm{m}), 4.79(1 \mathrm{H}, \mathrm{d}$, $J=7.8 \mathrm{~Hz}), 5.44-5.52(3 \mathrm{H}, \mathrm{m}), 5.83(1 \mathrm{H}, \mathrm{dd}, J=9.6,9.6 \mathrm{~Hz}), 7.24-7.30$ $(2 \mathrm{H}, \mathrm{m}), 7.34-7.44(5 \mathrm{H}, \mathrm{m}), 7.48-7.54(2 \mathrm{H}, \mathrm{m}), 7.80-7.84(2 \mathrm{H}, \mathrm{m})$, $7.90-7.98(4 \mathrm{H}, \mathrm{m}) ;{ }^{13} \mathrm{C}-\mathrm{NMR}\left(\mathrm{CDCl}_{3}\right): \delta-4.87,-4.82,18.8,21.6$ $26.3[3 \mathrm{C}], 31.7,31.9,38.2,39.2,41.2,43.7,63.4,70.4,72.3,72.6,73.8$, $75.8,100.4,121.5,128.7[2 \mathrm{C}], 128.7[2 \mathrm{C}], 128.8[2 \mathrm{C}], 129.5,129.7,130.0$, $130.2[2 \mathrm{C}], 130.3[2 \mathrm{C}], 130.3[2 \mathrm{C}], 133.5,133.6,133.7,144.6,165.6,165.7$, 166.4; HR-FAB-MS (NBA) $m / z$ : Calcd for $\mathrm{C}_{43} \mathrm{H}_{53} \mathrm{O}_{9} \mathrm{Si}: 741.3459(\mathrm{M}+1)^{+}$, Found: 741.3499

Myrtenyl 2,3,4-Tri- $\boldsymbol{O}$-benzoyl- $\boldsymbol{\beta}$-D-glucopyranoside (7) To a solution of $6(750 \mathrm{mg}, 1.01 \mathrm{mmol})$ in THF $(5 \mathrm{ml})$ was added $1 \mathrm{~N} \mathrm{HCl}(2.0 \mathrm{ml}$, $2.0 \mathrm{mmol}$ ) and stirred for $1 \mathrm{~h}$ at $\mathrm{rt}$. The reaction mixture was extracted with AcOEt. The organic layer was washed with $\mathrm{H}_{2} \mathrm{O}$, brine, dried over $\mathrm{Na}_{2} \mathrm{SO}_{4}$ and evaporated to give a residue, which was purified by flash column chromatography on silica gel $\{30 \mathrm{~g}, n$-hexane/AcOEt $(8: 1-2: 1)\}$ to afford 7 (501 mg, $0.8 \mathrm{mmol}, 79 \%)$ as a colorless oil. $7:[\alpha]_{\mathrm{D}}^{29}-25.6^{\circ}(c=0.77$, $\mathrm{CHCl}_{3}$ ); IR (KBr): 3509, 2937, 1733, 1602, 1451, $1093 \mathrm{~cm}^{-1} ;{ }^{1} \mathrm{H}-\mathrm{NMR}$ $\left(\mathrm{CDCl}_{3}\right): \delta 0.76(3 \mathrm{H}, \mathrm{s}), 1.04-1.08(1 \mathrm{H}, \mathrm{m}), 1.04(3 \mathrm{H}, \mathrm{s}), 1.95-2.20(2 \mathrm{H}$ m), $2.10-2.26(3 \mathrm{H}, \mathrm{m}), 3.71-3.90(3 \mathrm{H}, \mathrm{m}), 3.98-4.03(1 \mathrm{H}, \mathrm{m}), 4.23-$ $4.29(1 \mathrm{H}, \mathrm{m}), 4.84(1 \mathrm{H}, \mathrm{d}, J=7.8 \mathrm{~Hz}), 5.44-5.48(1 \mathrm{H}, \mathrm{m}), 4.48-5.55(2 \mathrm{H}$, m), $5.92(1 \mathrm{H}, \mathrm{dd}, J=9.6,9.6 \mathrm{~Hz}), 7.25-7.31(2 \mathrm{H}, \mathrm{m}), 7.36-7.45(5 \mathrm{H}, \mathrm{m})$, $7.49-7.56(2 \mathrm{H}, \mathrm{m}), 7.82-7.86(2 \mathrm{H}, \mathrm{m}), 7.93-7.98(4 \mathrm{H}, \mathrm{m}) ;{ }^{13} \mathrm{C}-\mathrm{NMR}$ $\left(\mathrm{CDCl}_{3}\right): \delta 21.5,26.3,31.7,31.9,38.2,41.2,43.7,61.9,70.2,72.4,73.0$, $73.3,75.1,100.9,121.5,128.8[4 \mathrm{C}], 129.0[2 \mathrm{C}], 129.2,129.4,130.0$ 130.2[2C], 130.3[2C], 130.4[2C], 133.6, 133.7, 134.1, 144.6, 165.5, 166.4, 166.5; HR-FAB-MS (NBA) $m / z$ : Calcd for $\mathrm{C}_{37} \mathrm{H}_{39} \mathrm{O}_{9}: 627.2594(\mathrm{M}+1)^{+}$, Found: 627.2563 .

Myrtenyl 2,3,4,2',3', $4^{\prime}$ - $O$-Hexabenzoyl- $\alpha$-L-arabinopyranosyl- $\beta$-D-glucopyranoside (9) To a solution of $7(335 \mathrm{mg}, 0.535 \mathrm{mmol})$ and 2,3,4-tri- $O$ benzoyl- $\alpha$-L-arabinopyranosyl bromide $(8,561 \mathrm{mg}, 1.07 \mathrm{mmol})$ in $\mathrm{CH}_{2} \mathrm{Cl}_{2}$ $(10 \mathrm{ml})$ was added tetramethylurea (TMU, $137 \mathrm{mg}, 1.18 \mathrm{mmol})$ at $0{ }^{\circ} \mathrm{C}$ under nitrogen atmosphere. AgOTf $(275 \mathrm{mg}, 1.07 \mathrm{mmol})$ was added to this reaction mixture at $0{ }^{\circ} \mathrm{C}$ under nitrogen atmosphere. The whole was covered with aluminum foil and stirred for $16 \mathrm{~h}$ at $\mathrm{rt}$. The reaction mixture was cooled to $0{ }^{\circ} \mathrm{C}$ and quenched with $7 \%$ aqueous $\mathrm{NaHCO}_{3}$ solution $(20 \mathrm{ml})$. The organic layer was washed with brine and dried over $\mathrm{Na}_{2} \mathrm{SO}_{4}$. Evaporation of the organic solvent gave a residue, which was purified by flash column chromatography on silica gel $\{50 \mathrm{~g}, n$-hexane/AcOEt $(8: 1-2: 1)\}$ to afford 9
(472 mg, $0.403 \mathrm{mmol}, 74 \%$ ) as a colorless amorphous. 9: $[\alpha]_{\mathrm{D}}^{29}+33.9^{\circ}$ $\left(c=0.45, \mathrm{CHCl}_{3}\right)$; IR (KBr): 2937, 1733, 1602, 1451, 1261, $1093 \mathrm{~cm}^{-1} ;{ }^{1} \mathrm{H}-$ NMR $\left(\mathrm{CDCl}_{3}\right): \delta 0.69(3 \mathrm{H}, \mathrm{s}), 0.93-0.98(1 \mathrm{H}, \mathrm{m}), 0.95(3 \mathrm{H}, \mathrm{s}), 1.80-1.85$ $(1 \mathrm{H}, \mathrm{m}), 1.90-1.97(1 \mathrm{H}, \mathrm{m}), 2.05(1 \mathrm{H}, \mathrm{ddd}, J=5.6,5.6,8.6 \mathrm{~Hz}), 2.00$ $2.18(2 \mathrm{H}, \mathrm{m}), 3.66-3.71(1 \mathrm{H}, \mathrm{m}), 3.83(1 \mathrm{H}, \mathrm{dd}, J=7.3,11.1 \mathrm{~Hz}), 3.83-$ $3.91(2 \mathrm{H}, \mathrm{m}), 3.98-4.05(1 \mathrm{H}, \mathrm{m}), 4.08(1 \mathrm{H}, \mathrm{dd}, J=1.8,11.1 \mathrm{~Hz}), 4.27(1 \mathrm{H}$, dd, $J=4.3,12.6 \mathrm{~Hz}), 4.69(1 \mathrm{H}, \mathrm{d}, J=8.1 \mathrm{~Hz}), 4.84(1 \mathrm{H}, \mathrm{d}, J=6.1 \mathrm{~Hz}), 5.26-$ $5.30(1 \mathrm{H}, \mathrm{m}), 5.38(1 \mathrm{H}, \mathrm{dd}, J=9.6,9.6 \mathrm{~Hz}), 5.41(1 \mathrm{H}, \mathrm{dd}, J=8.1,9.6 \mathrm{~Hz})$, $5.60(1 \mathrm{H}, \mathrm{dd}, J=3.5,8.6 \mathrm{~Hz}), 5.64-5.69(1 \mathrm{H}, \mathrm{m}), 5.73(1 \mathrm{H}, \mathrm{dd}, J=6.1$, $8.6 \mathrm{~Hz}), 5.80(1 \mathrm{H}, \mathrm{dd}, J=9.6,9.6 \mathrm{~Hz}), 7.23-7.28(2 \mathrm{H}, \mathrm{m}), 7.32-7.45$ $(11 \mathrm{H}, \mathrm{m}), 7.46-7.59(5 \mathrm{H}, \mathrm{m}), 7.76-7.80(2 \mathrm{H}, \mathrm{m}), 7.88-7.96(6 \mathrm{H}, \mathrm{m})$, $7.99-8.04(4 \mathrm{H}, \mathrm{m}) ;{ }^{13} \mathrm{C}-\mathrm{NMR}\left(\mathrm{CDCl}_{3}\right): \delta 21.1,25.7,31.1,31.4,37.6,40.6$, $43.1,62.3,68.2,68.4,69.8,69.9,70.3,71.9[2 \mathrm{C}], 73.0,73.8,100.0,100.8$, $121.4,128.2[4 \mathrm{C}], 128.4[2 \mathrm{C}], 128.4[6 \mathrm{C}], 128.8,128.9,129.1,129.3,129.4$, $129.4, \quad 129.7[2 \mathrm{C}], \quad 129.8[2 \mathrm{C}], \quad 129.9[6 \mathrm{C}], \quad 129.9[2 \mathrm{C}], 133.1, \quad 133.1$, 133.3[3C], 133.4, 143.6, 164.9, 165.2, 165.3, 165.5, 165.6, 165.8; HR-FABMS (NBA) $m / z$ : Calcd for $\mathrm{C}_{63} \mathrm{H}_{59} \mathrm{O}_{16}: 1071.3803(\mathrm{M}+1)^{+}$, Found: 1071.3812.

Myrtenyl 6- $O$ - $\alpha$-L-Arabinopyranosyl- $\beta$-D-glucopyranoside (Sacranoside A) (1) A mixture of $9(200 \mathrm{mg}, 0.187 \mathrm{mmol})$ and $\mathrm{NaOMe}(20 \mathrm{mg}$, $0.37 \mathrm{mmol})$ in MeOH-THF $(5: 1 ; 6 \mathrm{ml})$ was stirred for $1 \mathrm{~h}$ at $\mathrm{rt}$. The reaction mixture was condensed to give a residue, which was purified by flash column chromatography on silica gel $\left\{10 \mathrm{~g}, \mathrm{CHCl}_{3} / \mathrm{MeOH}(20: 1-5: 1)\right\}$ to afford $\mathbf{1}(37 \mathrm{mg}, 0.083 \mathrm{mmol}, 44 \%)$ as a colorless amorphous. 1: $[\alpha]_{\mathrm{D}}^{28}-52.2^{\circ}$ $(c=1.55, \mathrm{MeOH}) ; \mathrm{IR}(\mathrm{KBr}): 3380,2912,1652,1368,1078 \mathrm{~cm}^{-1} ;{ }^{1} \mathrm{H}-\mathrm{NMR}$ $\left(\mathrm{CD}_{3} \mathrm{OD}\right): \delta 0.87(3 \mathrm{H}, \mathrm{s}), 1.19(1 \mathrm{H}, \mathrm{d}, J=8.6 \mathrm{~Hz}), 1.30(3 \mathrm{H}, \mathrm{s}), 2.06-2.12$ $(1 \mathrm{H}, \mathrm{m}), 2.21-2.35(3 \mathrm{H}, \mathrm{m}), 2.42(1 \mathrm{H}, \mathrm{ddd}, J=5.6,5.6,8.6 \mathrm{~Hz}), 3.17-$ $3.22(1 \mathrm{H}, \mathrm{m}), 3.33-3.44(3 \mathrm{H}, \mathrm{m}), 3.51-3.56(2 \mathrm{H}, \mathrm{m}), 3.59(1 \mathrm{H}, \mathrm{dd}$, $J=6.6,8.8 \mathrm{~Hz}), 3.73(1 \mathrm{H}, \mathrm{dd}, J=5.3,11.4 \mathrm{~Hz}), 3.79-3.82(1 \mathrm{H}, \mathrm{m}), 3.87$ $(1 \mathrm{H}, \mathrm{dd}, J=3.3,12.4 \mathrm{~Hz}), 4.00(1 \mathrm{H}, \mathrm{dd}, J=1.5,12.4 \mathrm{~Hz}), 4.08(1 \mathrm{H}, \mathrm{dd}$, $J=2.0,11.4 \mathrm{~Hz}), 4.20(1 \mathrm{H}, \mathrm{dd}, J=1.5,12.4 \mathrm{~Hz}), 4.28(1 \mathrm{H}, \mathrm{d}, J=7.8 \mathrm{~Hz})$, $4.32(1 \mathrm{H}, \mathrm{d}, J=6.6 \mathrm{~Hz}), 5.56-5.58(1 \mathrm{H}, \mathrm{m}) ;{ }^{13} \mathrm{C}-\mathrm{NMR}\left(\mathrm{CD}_{3} \mathrm{OD}\right): \delta 21.6$, $26.6,32.2,32.5,38.9,42.2,44.5,66.7,69.4,69.5,71.6,72.4,72.9,74.2$, 75.0, 76.9, 78.0, 103.4, 105.1, 121.0, 146.3; HR-FAB-MS (NBA) $\mathrm{m} / \mathrm{z}$ : Calcd for $\mathrm{C}_{21} \mathrm{H}_{35} \mathrm{O}_{10}$ : $447.2228(\mathrm{M}+1)^{+}$, Found: 449.2229 .

Neryl 6-O-tert-Butyldimethylsilyl- $\beta$-D-glucopyranoside (11) A mixture of 10 (738 mg, $2.33 \mathrm{mmol})$, DMAP (14 $\mathrm{mg}, 0,117 \mathrm{mmol})$ and TBDM$\mathrm{SCl}(370 \mathrm{mg}, 2.45 \mathrm{mmol})$ in pyridine $(15 \mathrm{ml})$ was stirred for $14 \mathrm{~h}$ at $\mathrm{rt}$. The reaction mixture was evaporated under reduced pressure to give a residue, which was purified by flush column chromatography on silica gel $\{30 \mathrm{~g}, n$ hexane/AcOEt $(8: 1-2: 1)\}$ to afford $\mathbf{1 1}(807 \mathrm{mg}, 1.87 \mathrm{mmol}, 80 \%)$ as a white amorphous. 11: $[\alpha]_{\mathrm{D}}^{29}-31.0^{\circ}\left(c=3.3, \mathrm{CHCl}_{3}\right)$; IR (KBr): 3370, 2929, $1461,1375,1051 \mathrm{~cm}^{-1},{ }^{1} \mathrm{H}-\mathrm{NMR}\left(\mathrm{CDCl}_{3}\right): \delta 0.10(3 \mathrm{H}, \mathrm{s}), 0.10(3 \mathrm{H}, \mathrm{s}), 0.91$ $(9 \mathrm{H}, \mathrm{s}), 1.60(3 \mathrm{H}, \mathrm{s}), 1.68(3 \mathrm{H}, \mathrm{s}), 1.76(3 \mathrm{H}, \mathrm{d}, J=0.8 \mathrm{~Hz}), 2.02-2.13(4 \mathrm{H}$, m), $3.32-3.40(2 \mathrm{H}, \mathrm{m}), 3.55-3.62(2 \mathrm{H}, \mathrm{m}), 3.83(1 \mathrm{H}, \mathrm{dd}, J=6.3,10.4 \mathrm{~Hz})$, $3.93(1 \mathrm{H}, \mathrm{dd}, J=5.0,10.4 \mathrm{~Hz}), 4.14(1 \mathrm{H}$, ddd, $J=0.8,7.8,11.6 \mathrm{~Hz}), 4.30$ $(1 \mathrm{H}$, ddd, $J=0.8,6.3,11.6 \mathrm{~Hz}), 4.31(1 \mathrm{H}, \mathrm{d}, J=7.6 \mathrm{~Hz}), 5.05-5.11(1 \mathrm{H}$, m), 5.33-5.38 (1H, m); ${ }^{13} \mathrm{C}-\mathrm{NMR}\left(\mathrm{CDCl}_{3}\right): \delta-5.01[2 \mathrm{C}], 18.2,18.7,24.0$, $26.2,26.3[3 \mathrm{C}], 27.2,32.6,65.4,65.8,73.7,74.0,74.5,76.8,101.6,121.0$, 124.2, 132.6, 142.4; HR-FAB-MS (NBA) $m / z$ : Calcd for $\mathrm{C}_{22} \mathrm{H}_{43} \mathrm{O}_{6} \mathrm{Si}$ : $431.2829(\mathrm{M}+1)^{+}$, Found: 431.2829 .

Neryl 2,3,4-Tri- $O$-benzoyl-6- $O$-tert-butyldimethylsilyl- $\beta$-D-glucopyranoside (12) To a solution of $\mathbf{1 1}(800 \mathrm{mg}, 1.86 \mathrm{mmol})$ in pyridine $(10 \mathrm{ml})$ was added benzoylchloride $(1.07 \mathrm{ml}, 9.26 \mathrm{mmol})$ at $0^{\circ} \mathrm{C}$, and the whole was stirred for $24 \mathrm{~h}$ at $\mathrm{rt}$. The reaction mixture was diluted with water and extracted with AcOEt. The organic layer was washed with $0.5 \mathrm{~N} \mathrm{HCl}, \mathrm{H}_{2} \mathrm{O}$ and brine. Then the organic layer was dried over $\mathrm{Na}_{2} \mathrm{SO}_{4}$ and evaporated to give a residue, which was purified by flash column chromatography on silica gel $\{30 \mathrm{~g}, n$-hexane/AcOEt $(16: 1-4: 1)\}$ to afford $12(1.35 \mathrm{~g}, 1.82 \mathrm{mmol}$, 98\%) as a colorless syrup. 12: $[\alpha]_{\mathrm{D}}^{27}+19.6^{\circ}\left(c=3.24, \mathrm{CHCl}_{3}\right)$; IR $(\mathrm{KBr})$ : 2931, 1732, 1453, 1260, $1096 \mathrm{~cm}^{-1} ;{ }^{1} \mathrm{H}-\mathrm{NMR}\left(\mathrm{CDCl}_{3}\right): \delta 0.02(6 \mathrm{H}, \mathrm{s}), 0.87$ $(9 \mathrm{H}, \mathrm{s}), 1.57(3 \mathrm{H}, \mathrm{s}), 1.66(3 \mathrm{H}, \mathrm{s}), 1.69(3 \mathrm{H}, \mathrm{d}, J=0.8 \mathrm{~Hz}), 2.00-2.08(4 \mathrm{H}$, m), 3.79-3.87 $(3 \mathrm{H}, \mathrm{m}), 4.23(1 \mathrm{H}$, ddd, $J=0.8,7.8,12.0 \mathrm{~Hz}), 4.29(1 \mathrm{H}$, ddd, $J=0.8,6.0,12.0 \mathrm{~Hz}), 4.80(1 \mathrm{H}, \mathrm{d}, J=7.8 \mathrm{~Hz}), 4.99-5.04(1 \mathrm{H}, \mathrm{m}), 5.19-$ $5.25(1 \mathrm{H}, \mathrm{m}), 5.44(1 \mathrm{H}, \mathrm{dd}, J=7.8,9.8 \mathrm{~Hz}), 5.48(1 \mathrm{H}, \mathrm{dd}, J=9.8,9.8 \mathrm{~Hz})$, $5.83(1 \mathrm{H}, \mathrm{dd}, J=9.8,9.8 \mathrm{~Hz}), 7.24-7.29(2 \mathrm{H}, \mathrm{m}), 7.34-7.50(5 \mathrm{H}, \mathrm{m})$, $7.48-7.53(2 \mathrm{H}, \mathrm{m}), 7.79-7.83(2 \mathrm{H}, \mathrm{m}), 7.90-7.97(4 \mathrm{H}, \mathrm{m}) ;{ }^{13} \mathrm{C}-\mathrm{NMR}$ $\left(\mathrm{CDCl}_{3}\right): \delta-4.90,-4.87,18.2,18.8,23.9,26.2,26.3[3 \mathrm{C}], 27.1,32.6,63.4$, $65.7,70.4,72.6,73.9,75.8,99.9,121.2,124.2,128.7[2 \mathrm{C}], 128.7[2 \mathrm{C}]$, $128.8[2 \mathrm{C}], 129.6,129.8,130.1,130.2[4 \mathrm{C}], 130.3[2 \mathrm{C}], 132.4,133.5,133.5$, 133.7, 142.1, 165.6, 165.6, 166.4 ; HR-FAB-MS (NBA) $m / z$ : Calcd for $\mathrm{C}_{43} \mathrm{H}_{55} \mathrm{O}_{9} \mathrm{Si}: 743.3615(\mathrm{M}+1)^{+}$, Found: 743.3624.

Neryl 2,3,4-Tri- $\boldsymbol{O}$-benzoyl- $\boldsymbol{\beta}$-D-glucopyranoside (13) To a solution of $12(1.30 \mathrm{~g}, 0.82 \mathrm{mmol})$ in THF $(20 \mathrm{ml})$ was added $1 \mathrm{~N} \mathrm{HCl}(4.0 \mathrm{ml}, 4 \mathrm{mmol})$ 
and stirred for $16 \mathrm{~h}$ at $\mathrm{rt}$. The reaction mixture was extracted with AcOEt. The organic layer was washed with $\mathrm{H}_{2} \mathrm{O}$, brine, dried over $\mathrm{Na}_{2} \mathrm{SO}_{4}$ and evaporated to give a residue, which was purified by flash column chromatography on silica gel $\{30 \mathrm{~g}, n$-hexane/AcOEt $(8: 1-2: 1)\}$ to afford $13(1.03 \mathrm{~g}$, $1.63 \mathrm{mmol}, 93 \%)$ as a colorless oil. 13: $[\alpha]_{\mathrm{D}}^{26}+21.6^{\circ}\left(c=2.38, \mathrm{CHCl}_{3}\right)$; IR (KBr): 3444, 2966, 1731, 1453, 1262, $1097 \mathrm{~cm}^{-1} ;{ }^{1} \mathrm{H}-\mathrm{NMR}\left(\mathrm{CDCl}_{3}\right): \delta 1.57$ $(3 \mathrm{H}, \mathrm{s}), 1.66(3 \mathrm{H}, \mathrm{s}), 1.70(3 \mathrm{H}, \mathrm{d}, J=1.0 \mathrm{~Hz}), 1.95-2.05(4 \mathrm{H}, \mathrm{m}), 2.48$ $2.55(1 \mathrm{H}, \mathrm{m}), 3.71-3.79(2 \mathrm{H}, \mathrm{m}), 3.81-3.89(1 \mathrm{H}, \mathrm{m}), 4.25-4.30(2 \mathrm{H}, \mathrm{m})$, $4.86(1 \mathrm{H}, \mathrm{d}, J=7.8 \mathrm{~Hz}), 5.00-5.04(1 \mathrm{H}, \mathrm{m}), 5.20-5.24(1 \mathrm{H}, \mathrm{m}), 5.48(1 \mathrm{H}$, dd, $J=9.8,9.8 \mathrm{~Hz}), 5.49(1 \mathrm{H}, \mathrm{dd}, J=7.8,9.8 \mathrm{~Hz}), 5.92(1 \mathrm{H}, \mathrm{dd}, J=9.8$, $9.8 \mathrm{~Hz}), 7.27-7.29(2 \mathrm{H}, \mathrm{m}), 7.33-7.42(5 \mathrm{H}, \mathrm{m}), 7.48-7.56(2 \mathrm{H}, \mathrm{m})$, $7.74-7.88(2 \mathrm{H}, \mathrm{m}), 7.80-7.90(4 \mathrm{H}, \mathrm{m}) ;{ }^{13} \mathrm{C}-\mathrm{NMR}\left(\mathrm{CDCl}_{3}\right): \delta 18.2,23.9$, $26.2,27.1,32.7,61.9,65.0,70.2,72.4,73.4,75.2,100.0,120.9,124.1$, $128.8[4 \mathrm{C}], \quad 129.0[2 \mathrm{C}], \quad 129.2, \quad 129.4, \quad 130.0, \quad 130.3[2 \mathrm{C}], \quad 130.3[2 \mathrm{C}]$, 130.4[2C], 132.7, 133.6, 133.7, 134.1, 142.5, 165.6, 166.4, 166.5; HR-FABMS (NBA) $m / z$ : Calcd for $\mathrm{C}_{37} \mathrm{H}_{41} \mathrm{O}_{9}: 629.2751(\mathrm{M}+1)^{+}$, Found: 629.2752 .

Neryl $2,3,4,2^{\prime}, 3^{\prime}, 4^{\prime}$ - $O$-Hexabenzoyl- $\alpha$-L-arabinopyranosyl- $\beta$-D-glucopyranoside (14) To a solution of $13(275 \mathrm{mg}, 0.437 \mathrm{mmol})$ and 2,3,4-tri$O$-benzoyl- $\alpha$-L-arabinopyranosyl bromide $(\mathbf{8}, 460 \mathrm{mg}, 0.874 \mathrm{mmol})$ in $\mathrm{CH}_{2} \mathrm{Cl}_{2}(10 \mathrm{ml})$ was added TMU $(111 \mathrm{mg}, 0.96 \mathrm{mmol})$ at $0{ }^{\circ} \mathrm{C}$ under nitrogen atmosphere. AgOTf $(225 \mathrm{mg}, 0.874 \mathrm{mmol})$ was added to this reaction mixture at $0{ }^{\circ} \mathrm{C}$ under a nitrogen atmosphere. The whole was covered with aluminum foil and stirred for $14 \mathrm{~h}$ at $\mathrm{rt}$. The reaction mixture was cooled to $0{ }^{\circ} \mathrm{C}$ and quenched with $7 \%$ aqueous $\mathrm{NaHCO}_{3}$ solution $(20 \mathrm{ml})$. The organic layer was washed with brine and dried over $\mathrm{Na}_{2} \mathrm{SO}_{4}$. Evaporation of the organic solvent gave a residue, which was purified by flash column chromatography on silica gel $\{20 \mathrm{~g}, n$-hexane/AcOEt $(8: 1-2: 1)\}$ to afford $\mathbf{1 4}$ $(452 \mathrm{mg}, 0.421 \mathrm{mmol}, 96 \%)$ as a colorless amorphous. 14: $[\alpha]_{\mathrm{D}}^{26}+69.5^{\circ}$ $\left(c=0.81, \mathrm{CHCl}_{3}\right)$; IR (KBr): 2934, 1729, 1453, 1261, $1095 \mathrm{~cm}^{-1} ;{ }^{1} \mathrm{H}-\mathrm{NMR}$ $\left(\mathrm{CDCl}_{3}\right): \delta 1.53(3 \mathrm{H}, \mathrm{d}, J=0.8 \mathrm{~Hz}), 1.61(3 \mathrm{H}, \mathrm{s}), 1.64(3 \mathrm{H}, \mathrm{s}), 1.87-2.00$ $(4 \mathrm{H}, \mathrm{m}), 3.82(1 \mathrm{H}, \mathrm{dd}, J=6.6,11.4 \mathrm{~Hz}), 3.85(1 \mathrm{H}, \mathrm{dd}, J=2.3,12.6 \mathrm{~Hz})$, $3.95-4.06(3 \mathrm{H}, \mathrm{m}), 4.10(1 \mathrm{H}, \mathrm{dd}, J=2.0,11.4 \mathrm{~Hz}), 4.26(1 \mathrm{H}, \mathrm{dd}, J=4.8$, $12.6 \mathrm{~Hz}), 4.73(1 \mathrm{H}, \mathrm{d}, J=7.8 \mathrm{~Hz}), 4.84(1 \mathrm{H}, \mathrm{d}, J=5.8 \mathrm{~Hz}), 4.94-5.00(1 \mathrm{H}$, $\mathrm{m}), 5.07-5.13(1 \mathrm{H}, \mathrm{m}), 5.37(1 \mathrm{H}, \mathrm{dd}, J=7.8,9.6 \mathrm{~Hz}), 5.41(1 \mathrm{H}, \mathrm{dd}, J=9.2$, $9.6 \mathrm{~Hz}), 5.61(1 \mathrm{H}, \mathrm{dd}, J=3.5,8.1 \mathrm{~Hz}), 5.63-5.67(1 \mathrm{H}, \mathrm{m}), 5.71(1 \mathrm{H}, \mathrm{dd}$, $J=5.8,8.1 \mathrm{~Hz}), 5.81(1 \mathrm{H}, \mathrm{dd}, J=9.6,9.6 \mathrm{~Hz}), 7.23-7.28(2 \mathrm{H}, \mathrm{m}), 7.31-$ $7.59(16 \mathrm{H}, \mathrm{m}), 7.75-7.79(2 \mathrm{H}, \mathrm{m}), 7.86-7.94(4 \mathrm{H}, \mathrm{m}), 7.95-8.04(6 \mathrm{H}$, $\mathrm{m}) ;{ }^{13} \mathrm{C}-\mathrm{NMR}\left(\mathrm{CDCl}_{3}\right): \delta 18.1,23.8,26.2,27.1,32.4,62.3,65.6,68.6[2 \mathrm{C}]$, $70.2,70.3,70.6,72.4,73.6,74.3,100.0,101.1,120.7,124.2,128.7[2 \mathrm{C}]$, $128.7[2 \mathrm{C}], 128.8[2 \mathrm{C}], 128.9[4 \mathrm{C}], 129.0[2 \mathrm{C}], 129.3,129.4,129.7,129.8$, $129.9,130.0,130.2[2 \mathrm{C}], 130.3[2 \mathrm{C}], 130.3[4 \mathrm{C}], 130.4[4 \mathrm{C}], 132.3,133.5$, $133.6,133.8,133.8,133.9[2 \mathrm{C}], 142.7,165.5,165.6,165.7,166.0,166.1$, 166.3; HR-FAB-MS (NBA) $m / z$ : Calcd for $\mathrm{C}_{63} \mathrm{H}_{61} \mathrm{O}_{16}: 1073.3959(\mathrm{M}+1)^{+}$, Found: 1073.3994.
Neryl 6- $\boldsymbol{O}$ - $\boldsymbol{\alpha}$-L-Arabinopyranosyl- $\boldsymbol{\beta}$-D-glucopyranoside (Sacranoside B) (2) A mixture of $\mathbf{1 4}(200 \mathrm{mg}, 0.186 \mathrm{mmol})$ and $\mathrm{NaOMe}(20 \mathrm{mg}$, $0.37 \mathrm{mmol})$ in $\mathrm{MeOH}-\mathrm{THF}(1: 1 ; 10 \mathrm{ml})$ was stirred for $3 \mathrm{~h}$ at $\mathrm{rt}$. The reaction mixture was condensed to give a residue, which was purified by flash column chromatography on silica gel $\left\{10 \mathrm{~g}, \mathrm{CH}_{2} \mathrm{Cl}_{2} / \mathrm{MeOH}(9: 1-5: 1)\right\}$ to afford $2(40 \mathrm{mg}, 0.09 \mathrm{mmol}, 48 \%)$ as a colorless amorphous. 2 : $[\alpha]_{\mathrm{D}}^{26}-34.9^{\circ}$ $(c=1.85, \mathrm{MeOH}) ; \mathrm{IR}(\mathrm{KBr}): 3379,2921,1659,1444,1258 \mathrm{~cm}^{-1} ;{ }^{1} \mathrm{H}-\mathrm{NMR}$ $\left(\mathrm{CD}_{3} \mathrm{OD}\right): \delta 1.61(3 \mathrm{H}, \mathrm{s}), 1.68(3 \mathrm{H}, \mathrm{s}), 1.75(3 \mathrm{H}, \mathrm{d}, J=1.0 \mathrm{~Hz}), 2.04-2.16$ $(4 \mathrm{H}, \mathrm{m}), 3.18(1 \mathrm{H}, \mathrm{dd}, J=7.8,9.0 \mathrm{~Hz}), 3.33-3.39(3 \mathrm{H}, \mathrm{m}), 3.52(1 \mathrm{H}, \mathrm{dd}$, $J=3.5,8.8 \mathrm{~Hz}), 3.53(1 \mathrm{H}, \mathrm{dd}, J=1.8,12.4 \mathrm{~Hz}), 3.60(1 \mathrm{H}, \mathrm{dd}, J=6.6$, $8.8 \mathrm{~Hz}), 3.74(1 \mathrm{H}, \mathrm{dd}, J=4.5,11.4 \mathrm{~Hz}), 3.79-3.82(1 \mathrm{H}, \mathrm{m}), 3.87(1 \mathrm{H}, \mathrm{dd}$, $J=3.3,12.4 \mathrm{~Hz}), 4.08(1 \mathrm{H}, \mathrm{dd}, J=1.7,11.4 \mathrm{~Hz}), 4.17(1 \mathrm{H}, \mathrm{dd}, J=7.6$, $11.4 \mathrm{~Hz}), 4.27(1 \mathrm{H}, \mathrm{d}, J=7.8 \mathrm{~Hz}), 4.31(1 \mathrm{H}, \mathrm{d}, J=6.6 \mathrm{~Hz}), 4.28-4.35(1 \mathrm{H}$, $\mathrm{m}), 5.09-5.15(1 \mathrm{H}, \mathrm{m}), 5.35-5.41(1 \mathrm{H}, \mathrm{m}) ;{ }^{13} \mathrm{C}-\mathrm{NMR}\left(\mathrm{CD}_{3} \mathrm{OD}\right): \delta 17.8$, 23.7, 25.9, 27.7, 33.1, 66.4, 66.6, 69.3, 69.4, 71.5, 72.3, 74.2, 75.0, 76.7, $77.9,103.1,105.1,122.5,125.0,132.8,141.8$; HR-FAB-MS (NBA) $m / z$ : Calcd for $\mathrm{C}_{21} \mathrm{H}_{36} \mathrm{NaO}_{10}: 471.2206(\mathrm{M}+\mathrm{Na})^{+}$. Found: 471.2213

Acknowledgement The authors are grateful to Prof. M. Yoshikawa at Kyoto Pharmaceutical University for generously confirming the spectral data $\left({ }^{1} \mathrm{H}\right.$ - and $\left.{ }^{13} \mathrm{C}-\mathrm{NMR}\right)$ and optical rotations of natural products $\mathbf{1}$ and $\mathbf{2}$.

\section{References and Notes}

1) Francis M. J. O., Allcock C., Phytochemistry, 8, 1339-1347 (1969).

2) Yoshikawa M., Shimada Hirom. Horikawa S., Murakami T., Shimada Hiros., Yamahara J., Matsuda H., Chem. Pharm. Bull., 45, 1498-1503 (1997).

3) Fan W., Tezuka Y., Komatsu K., Namba T., Kadota S., Biol. Pharm. Bull., 22, 157-161 (1999).

4) Mook-Jung I., Kim H., Fan W., Tezuka Y., Kadota S., Nishijo H., Jung M. W., Biol. Pharm. Bull., 25, 1101-1104 (2002).

5) Kimura T., Jyo M., Nakamura N., Komatsu K., Hattori M., Shimotohno K., Kakiuchi N., J. Trad. Med., 20, 243-250 (2003).

6) Vic G., Crout D. H. G., Tetrahedron: Asymmetry, 5, 2513-2516 (1994).

7) Akita H., Kawahara E., Kato K., Tetrahedron: Asymmetry, 15, 1623 1629 (2004).

8) Hanessian S., Banoub J., Carbohydr. Res., 53, C13-C16 (1977).

9) Fletcher H. G., Hudson C. S., Jr., J. Am. Chem. Soc., 69, 1145-1147 (1947).

10) According to a private communication from Prof. M. Yoshikawa, the opposite sign of the reported optical rotation of natural 2 in comparison to that of synthetic 2 was presumably attributed to contamination by a small amount of impurity. 